auch bei peripheren Entzündungen im Gelenk nachgewiesen werden. Auch Synovitis und Knochendestruktion können durch parasympathisch vermittelte geringere Zytokinbildung gebremst werden, was die Hypothese einer endogenen suppressiven Aktivität von Entzündungen unterstützt.

Schaibles eigene Untersuchungen zur Bedeutung von Makrophagen für den
Schmerz und die Nozizeption ergaben, dass Makrophagen bei Entzündungen im Kniegelenk in die „dorsal root“-Ganglien einwandern. Solche MakrophagenCluster sehe man auch bei neuropathischen Störungen. Die noch zu überprüfende Hypothese ist, dass dies der Vermittlung parasympathischer Effekte in entzündetes Gewebe dient. Diese Vagusaktivität könnte - laut einer weiteren
Hypothese [Tracey KJ. J Clin Invest 2007; 117: 289 - 96] - durch Akupunktur, Biofeedback oder Sport unterstützt werden, was allerdings bislang klinisch kaum erforscht ist.

Dr. Andreas Häckel

"Das vegetative Nervensystem und Schmerz: Mögliche Zusammenhänge und therapeutische Optionen." Deutscher Schmerzkongress, Mannheim, 22.10.2016

\section{Nicht nur der Trigeminus steckt hinter Neuralgien}

\author{
Kopfschmerz und HNO-Symptome wie Phonophobie, Schwindel, Tinnitus, \\ Hörstörungen oder Dysphagie/Odynophagie gehen viel häufiger als ange- \\ nommen Hand in Hand.
}

D a oft zu lange gewartet werde, um in der Bildgebung noch etwas zu finden, sei es für die Klassifikation von Kopf- und Gesichtsschmerzen mit HNO-Komponente wichtig, die Zeitdauer zu klären, betonte Privatdozent Dr. Tim Jürgens, Klinik für Neurologie, Kopfschmerzzentrum Nord-Ost, Universitätsmedizin Rostock. Daher sei eine gute Anamnese wichtig. Als wichtigste Neuralgien mit HNO-Komponente nannte Jürgens die Glossopharyngeus-, Intermedius- und Occipitalis-Neuralgie. Erstere imponiert durch Schmerzen im hinteren Teil von Zunge, Tonsillen und Pharynx unterhalb des Unterkiefers und/oder im Ohr. Der Schmerz ist ste-

chend, einschießend und von hoher Intensität. Weitere Symptome sind Paroxysmen von wenigen Sekunden bis zwei Minuten Dauer, selten auch kardiale Symptome (Synkopen, Asystolie) und in $10 \%$ eine Trigeminusneuralgie. Die Neuralgie wird durch Schlucken, Husten, Sprechen oder Gähnen getriggert. Ursächlich sei eine Reizung von Ästen der N. glossopharyngeus und vagus durch Gefäß-Nerven-Kontakt.

Therapie der Wahl sind topische Lokalanästhetika. Alternativ kommen Carbamazepin/Oxcarbazepin infrage, für die Langzeittherapie Lamotrigin, Phenytoin i.v. und eventuell eine neurovaskuläre Dekompression, sagte Jürgens.

\section{Gene und multikulturelle Aspekte}

In der schmerztherapeutischen Versorgung von Migranten gibt es konkrete biologische und genetische Faktoren zu berücksichtigen. Dazu zählen vor allem Genpolymorphismen und -mutationen, welche die Metabolisierung von Medikamenten beeinflussen und damit auch deren Wirkung und Nebenwirkungsprofil. Wie Professor Ulrike Stamer, Klinik für Anästhesiologie und Schmerztherapie des Inselspitals der Universität Bern, berichtete, können „Poor metabolizer" für CYP2D6, die im Mittelmeerraum häufiger sind, Codein und Tramadol nicht in die aktiven Metaboliten umwandeln. Sie weisen dadurch keine oder eine reduzierte
Analgesie auf. Umgekehrt kann es bei "ultra rapid metabolizern", die in Ostafrika, Äthiopien, dem Nahen Osten und Ozeanien fast ein Drittel der Bevölkerung ausmachen, zu einer Atemdepression wie bei einer Überdosierung kommen. Es sei wichtig, dies im Kopf zu behalten, wenn der Erfolg der Schmerztherapie evaluiert Testung auf Genotypen wird es so bald nicht geben. Dr. Wiebke Kathmann

„Multikulturelle Aspekte der Schmerztherapie: (Um)Denken erwünscht". Deutscher Schmerzkongress, Mannheim, 22.10.2016 werde, so Stamer. Denn eine genetische
Schmerzqualitäten, Begleitsymptome und Therapieoptionen bei IntermediusNeuralgie ähneln denen bei Glossopharyngeus-Neuralgie. Die Schmerzen betreffen hier den Gehörgang, teils auch parietal, und werden durch Berührung der hinteren Wand des Gehörgangs und/ oder periaurikulär getriggert. Da mehrere Nerven beteiligt sind, ist ein Mischbild möglich. Bei der Occipitalis-Neuralgie treten die uni- oder bilateralen Schmerzen im Ausbreitungsgebiet des N. occipitalis major, minor oder tertius auf. Neben Paroxysmen finden sich Dysästhesie und/oder Allodynie bei schmerzloser Stimulation der Haut und ein Spannungsgefühl der betroffenen Haut oder schmerzhafte Druckpunkte über dem Nervenverlauf.

\section{Neues zu trigemino-autonomen Kopfschmerzen}

Wie Jürgens berichtete, ist in der Leitlinien von $2016 \mathrm{zu}$ trigemino-autonomen Kopfschmerzen ein autonomes Symptom hinzugekommen - das Völlegefühl im Ohr. Neu ist auch die vestibuläre Migräne, bei der die vestibulären Symptome in Form eines Lagerungs- oder zentralen Schwindels etwas kürzer andauern als die Migräne. Gerade wenn es sich um eine Migräne mit Aura handele, könne diese mit weiteren seltsamen Symptomen vergesellschaftet sein, so Jürgens. Dazu zählen visuelle Illusionen, die attackenartig auftreten oder persistieren, visuelle Halluzinationen, Dyschromatopsie sowie Synästhesien. Flunarizin ist hier Mittel der ersten Wahl.

Dr. Wiebke Kathmann

"Schwerpunkt Kopfschmerz: DMKG meets HNO - relevante Schmerzsyndrome von Hals, Nase und Ohr", Deutscher Schmerzkongress, Mannheim, 22.10.2016 